\title{
Low prevalence of methicillin-resistant Staphylococcus aureus (MRSA) carriage in women from former Yugoslavia living in Switzerland
}

\author{
J. Marschall • P. Dürig • K. Mühlemann
}

Published online: 18 July 2006

(C) Springer-Verlag 2006

Methicillin-resistant Staphylococcus aureus (MRSA) carriage may be more frequent in individuals originating from countries known to have a high MRSA prevalence. In the study presented here, the prevalence of MRSA carriers among healthy pregnant women from former Yugoslavia living in Switzerland was evaluated by surveillance of screening cultures. Among 152 women screened, no MRSA carrier was detected $(95 \%$ confidence interval for carriage prevalence, $0-1.9 \%$ ). Currently, specific infection control measures such as routine screening for MRSA at hospital admission do not seem necessary in this patient population.

In recent years, methicillin-resistant Staphylococcus aureus (MRSA) strains have been recognized in outpatients and are now known as community-acquired MRSA (CA-MRSA). These strains differ from hospital-acquired (HA-MRSA) strains. CA-MRSA strains are being reported from an increasing number of countries, including those with a low prevalence of HA-MRSA. At the University Hospital Bern, the prevalence of HA-MRSA is below 5\%,

This data was presented in part at the Annual Assembly of the Swiss Society for Infectious Diseases, Basel, Switzerland, 6-8 June 2005.

J. Marschall $\cdot$ K. Mühlemann $(\bowtie)$

Division of Infectious Diseases, University Hospital Bern,

Friedbühlstrasse 51,

3010 Bern, Switzerland

e-mail: kathrin.muehlemann@ifik.unibe.ch

P. Dürig

Department of Obstetrics and Gynaecology,

University Hospital Bern,

Bern, Switzerland

P. Dürig

Effinger-Zentrum for Obstetrics and Gynecology,

Effingerstrasse 453008 Bern, Switzerland and routine screening for MRSA carriage is restricted to inpatients and patients referred from a foreign hospital. However, in recent months several cases of vaginal MRSA carriage have been detected by chance in women giving birth (unpublished observation). It is noteworthy that a high percentage of these women were originally from former Yugoslavia. In countries such as Croatia, the prevalence of HA-MRSA has been reported to be $22 \%$ [1], but no data have yet been published on the occurrence of CA-MRSA in that country.

Between 12 January 2004 and 5 January 2005 we performed a screening study to evaluate MRSA carriage in pregnant women from former Yugoslavia at the obstetrical outpatient clinic of the University Hospital Bern, a tertiary care center with more than 1,000 births per year. The study was performed in accordance with the guidelines of the local ethical committee. During the study period, each woman was screened once for MRSA carriage at an antenatal check.

Screening was performed by the treating physician in accordance with written in-house guidelines. Using twisted wire rayon-tipped applicators (Copan Venturi Transystem; Copan, Brescia, Italy) premoistened with physiological saline, nasal swabs were taken from both nares (same applicator) and vaginal swabs were taken from the introitus vaginae, including the perineal region. Microbiological screening and identification of MRSA were performed using the mannitol/oxacillin biplate test, selective culture for grampositive organisms, and the coagulase tube test for exclusion of coagulase-negative Staphylococcus spp; antimicrobial resistance testing was performed using Müller-Hinton agar. Patients' data were collected from clinical charts and hospital statistics using a standardized questionnaire. The 95\% confidence interval for MRSA prevalence was calculated based on a binomial distribution. 
A total of 152 women were screened for MRSA carriage, and no MRSA carrier was detected (confidence interval for carriage prevalence, $0-1.9 \%)$. Women presented either on their own initiative $(64 \%)$ or following referral from practitioners $(28 \%)$ or other hospitals $(9 \%)$. The mean age was 25.1 years $( \pm 4.93$, range $18-40$ years). The women originated mainly from Serbia, Montenegro and Kosovo (51\%) and Macedonia (32\%). About one-third (38\%) had immigrated to Switzerland since 2001 and 14 (9\%) had immigrated during the 12-month period preceding MRSA screening. About half of the women were housewives (54\%) and only a few worked in a healthcare setting (5\%). There were $78(51 \%)$ nulliparae women, 42 were primiparae $(28 \%)$ and $31(20 \%)$ had given birth two or more times previously. MRSA screening was performed at a mean of $233 / 7$ (range 7 0/7-40 4/7) weeks of pregnancy. At least 15 (10\%) women visited their home country during their pregnancy and eight $(5 \%)$ reported having a medical consultation while in their home country. During the 12 months before MRSA screening, $12(8 \%)$ women had had surgery; in eight of them this consisted of curettage for incomplete abortion. Antibiotic therapy was prescribed to $26(17 \%)$ women during pregnancy. Only four $(3 \%)$ were treated with a vaginally applied disinfectant (chlorhexidine).

Our results show the prevalence of MRSA carriage among women of childbearing age from former Yugoslavia was very low in this region of Switzerland. Screening of the genital tract for MRSA colonization has been rarely performed until now. Dancer and Noble [2] reported data on 500 pregnant women who were screened for Staphylococcus aureus using nasal, axillary and perineal swabs, and $33 \%$ were found to be carriers. However, that study did not indicate whether the three MRSA isolates detected were collected from the perineum. With respect to MRSA infections, a recently published case series showed that 12 of 57 pregnant women had an infection of the vulva or groin [3].

Few studies have examined the prevalence of MRSA in the Swiss community [4-6]; however, two surveys found varying patterns of geographical distribution of MRSA among drug users $[4,5]$. A recent study performed in Geneva in western Switzerland found CA-MRSA in roughly $0.1 \%$ of 14,000 patients screened at hospital admission [6]. This low prevalence of CA-MRSA is in accordance with several community-based screenings in other European states [7, 8]. However, recent investigations point to an increase of CA-MRSA in Europe [9].

MRSA carriage in otherwise healthy individuals without a relevant history of hospital contact has been shown in the case of Kosovan refugees in Denmark [10], a finding which probably reflects the earlier mentioned increase in MRSA prevalence in former Yugoslavia [1]. Yet, a familial link to a region with an impaired healthcare system does not seem to predict carriage of nosocomial pathogens, as our results indicate. Therefore, specific infection control measures such as routine screening for MRSA at hospital admission do not seem to be necessary in this patient population in Switzerland.

Acknowledgements We would like to thank L. Beul, S. Burri, S. Droz, R. Kuhn and T. Löhri for technical assistance. The study complied with current Swiss law.

\section{References}

1. Tambic Andrasevic A, Tambic T, Kalenic S, Jankovic V (2002) Surveillance for antimicrobial resistance in Croatia. Emerg Infect Dis 8:14-18

2. Dancer SJ, Noble WC (1991) Nasal, axillary, and perineal carriage of Staphylococcus aureus among women: identification of strains producing epidermolytic toxin. Pathol 44:681-684

3. Laibl VR, Sheffield JS, Roberts S, McIntire DD, Trevino S, Wendel GD (2005) Clinical presentation of community-acquired methicillin-resistant Staphylococcus aureus in pregnancy. Obstet Gynecol 106:461-465

4. Fleisch F, Zbinden R, Vanoli C, Ruef C (2001) Epidemic spread of a single clone of methicillin-resistant Staphylococcus aureus among injection drug users in Zurich, Switzerland. Clin Infect Dis 32:581-586

5. Bassetti S, Wolfisberg I, Jaussi B, Frei R, Kuntze MF, Battegay M, Widmer AF (2004) Carriage of Staphylococcus aureus among injection drug users: lower prevalence in an injection heroin maintenance program than in an oral methadone program. Infect Control Hosp Epidemiol 25:133-137

6. Harbarth S, François P, Schrenzel J, Fankhauser-Rodriguez C, Hugonnet S, Koessler T, Huyghe A, Pittet D (2005) Communityassociated methicillin-resistant Staphylococcus aureus, Switzerland. Emerg Inf Dis 11:962-965

7. Sa-Leao R, Sanches IS, Couto I, Alves CR, de Lencastre H (2001) Low prevalence of methicillin-resistant strains among Staphylococcus aureus colonizing young and healthy members of the community in Portugal. Microb Drug Resist 7:237-245

8. Abudu L, Blair I, Fraise A, Cheng KK (2001) Methicillin-resistant Staphylococcus aureus (MRSA): a community-based prevalence survey. Epidemiol Infect 126:351-356

9. Wannet WJ, Spalburg E, Heck ME, Pluister GN, Tiemersma E, Willems RJ, Huijsdens XW, de Neeling AJ, Etienne J (2005) Emergence of virulent methicillin-resistant Staphylococcus aureus strains carrying the Panton-Valentine leucocidin genes in the Netherlands. J Clin Microbiol 43:3341-3345

10. Hansen B, Pedersen LN, Arpi M, Obel N (2000) Incidence of methicillin-resistant Staphylococcus aureus among Kosovar-Albanian refugees at the refugee-center in Randers. Ugeskr Laeger 162:6241-6243 